\title{
Enhanced Supersymmetry of Nonrelativistic ABJM Theory
}

\author{
O-Kab Kwon ${ }^{1), *} \quad$ Phillial $\mathrm{Oh}^{1), \dagger} \quad$ Corneliu Sochichiu ${ }^{2), ;} \quad$ Jongsu Sohn ${ }^{1), \S}$
}

1) Department of Physics, BK21 Physics Research Division, Institute of Basic Science, Sungkyunkwan University, Suwon 440-746, Korea

2) University College, Sungkyunkwan University, Suwon 440-746, Korea and

Institutul de Fizică Aplicată AS, str. Academiei, nr. 5, Chişinău, MD2028 Moldova.

November 18, 2018

\begin{abstract}
We study the supersymmetry enhancement of nonrelativistic limits of the ABJM theory for Chern-Simons level $k=1,2$. The special attention is paid to the nonrelativistic limit (known as 'PAAP' case) containing both particles and antiparticles. Using supersymmetry transformations generated by the monopole operators, we find additional 2 kinematical, 2 dynamical, and 2 conformal supercharges for this case. Combining with the original 8 kinematical supercharges, the total number of supercharges becomes maximal: 14 supercharges, like in the well-known PPPP limit. We obtain the corresponding super Schrödinger algebra which appears to be isomorphic to the one of the PPPP case. We also discuss the role of monopole operators in supersymmetry enhancement and partial breaking of supersymmetry in nonrelativistic limit of the ABJM theory.
\end{abstract}

*email: okab@skku.edu

†email: ploh@skku.edu

‡email: sochichi@skku.edu

$\S$ email: jongsusohn@skku.edu 


\section{Introduction}

In the shed of recent development of M2-brane theories the three dimensional Chern-Simons based superconformal theories [1] gained a considerable interest. Two different types of theories were proposed as candidates to effectively describe multiple M2-branes system: the 3-algebra based BLG (Bagger-Lambert and Gustavsson) theory [2, 3] and standard Lie algebra based ABJM (Aharony-Bergman-Jafferis-Maldacena) theory [4]. The former theory is restricted to describe the effective action of two M2-branes only. As a result of this, lately the main interest focused on the ABJM and related theories, in which the setup with an arbitrary number of M2-branes is allowed. Beyond that, the ABJM theory proved to reproduce the correct vacuum structure of M2-branes on $\mathbb{C}^{4} / \mathbb{Z}_{k}$-orbifold, where $k$ appears as the Chern-Simons level in the theory.

The BLG theory, which is equivalent to the ABJM theory with $\mathrm{SU}(2) \times \mathrm{SU}(2)$ gauge group [4], has $\mathcal{N}=8$ supersymmetry (SUSY). On the other hand, the original ABJM theory has only manifest $\mathcal{N}=6$ supersymmetry instead of $\mathcal{N}=8$ expected from the M2-brane system in flat transverse space. The lack of supersymmetries can be explained by the fact that for the ChernSimons level $k$ the theory describes multiple M2-branes in $\mathbb{C}^{4} / \mathbb{Z}_{k}$-orbifold rather than in flat space and $\mathcal{N}=6$ is the maximal supersymmetry which the $\mathbb{C}^{4} / \mathbb{Z}_{k}$-orbifold allows, if $k \geq 3$. In what concerns the cases of $k=1,2$, it was conjectured from the dual gravity that the full $\mathcal{N}=8$ supersymmetry should be restored [4]. Indeed, using monopole operators, it was proved that the ABJM theory has additional $\mathcal{N}=2$ supersymmetry for $k=1,2$ [5, 6 . At these levels, attaching a monopole operator to a local field changes the rule of gauge transformation of the field, while preserving the local nature of the field 1 . Using this procedure we can introduce new fields in the theory with the help of monopole operators. In Ref. [5] the additional $\mathcal{N}=2$ supersymmetry and $\mathrm{SO}(8)$-invariance of the ABJM potential were obtained for $\mathrm{U}(N) \times \mathrm{U}(N)$ gauge group at $k=1,2$ in 3 -algebra formulation. The same enhancement of supersymmetry in the Lie-algebra formulation of ABJM theory for the case of $\mathrm{U}(2) \times \mathrm{U}(2)$ gauge group was found in Ref. [6], where the so called minimal model which inherits most properties of the ABJM theory was introduced. This minimal model can be used as a toy model replacement for the ABJM theory.

Recently there has been much interest in nonrelativistic versions of AdS/CFT correspondence [17 28]. The geometrical framework for nonrelativistic (super)symmetry was studied earlier in [29] (see also [30]). Following the development of superconformal field theories describing the dynamics of M2-branes, the construction of nonrelativistic superconformal field theories and finding their gravity duals become of increasing interest. In the past year, a number of nonrelativistic limits of the ABJM theory were constructed [31,32]. The nonrelativistic AdS/CFT correspondence [33 36] in relation with M-theory and the soliton solution [37] in nonrelativistic ABJM theory were also studied.

It is known that some nonrelativistic limits result in additional symmetries, which are not present in the original theory. Schrödinger symmetry [38] is such an example. Emergence of new

\footnotetext{
${ }^{1}$ For the detailed study of monopole operators in ABJM theory and related topics, see Refs. [7 16.
} 
bosonic symmetries can be accompanied by the emergence of new fermionic ones. However, there are not so many examples where the explicit construction of nonrelativistic superconformal field theories and analysis of their super Schrödinger algebras were done [39 42].

In this paper we find additional 6 supercharges using the monopole operators and complete the corresponding super Schrödinger algebra in a nonrelativistic limit of the ABJM theory, referred in Ref. [31] as 'PAAP limit2.

In general, the nonrelativistic limit of a field theory is ambiguous and depends on the particleantiparticle sectors chosen. The supersymmetry of various nonrelativistic limits of the ABJM theory was studied by several authors in [31,32]. These authors studied supersymmetry and super Schrödinger algebras for different non-relativistic regimes. In particular, it was found that one can define non-relativistic limits in which different numbers of supersymmetries are conserved [31]. The 'basic' $\mathcal{N}=6$ supersymmetry in the mass deformed ABJM theory [43, 44] survives and resulting 12 supercharges are split into 10 kinematical and 2 dynamical ones in the limit which involves only particles (PPPP limit). Additional 2 supercharges emerge coming from conformal symmetry associated with different scalings of time and space. However, if we include antiparticle sectors, the number of supersymmetry is reduced and superconformal symmetry is broken [31]. This is considered as a general property of the nonrelativistic limits of superconformal field theories [41, 42.

Our aim in this work is to extend the analysis to supersymmetry driven by monopole operators. In nonrelativistic limit where both particles and antiparticles are included e.g. PAAP limit, it appears that some of the supersymmetry can be restored. In order to have the enhanced supersymmetry, we should restrict ourself to the Chern-Simons levels $k=1,2$. Although the most analysis is done for the gauge group $\mathrm{U}(2) \times \mathrm{U}(2)$, we believe our conclusions hold true for the general case of $\mathrm{U}(N) \times \mathrm{U}(N)$.

The plan of the paper is as follows. In the next section we review the mass deformation and nonrelativistic limits of the ABJM theory following [31]. In the third section we introduce the PAAP limit and describe the known 8 kinematical supercharges as well as bosonic symmetries. In the fourth section we analyze the enhanced symmetry generated by monopole operator. We find that in the PPPP limit with maximal basic supersymmetry $Q_{1}+Q_{2}+S=10+2+2$, the monopole enhanced part of supersymmetry [5, 6] is broken 3 . In contrast, in the PAAP limit, where the basic nonrelativistic symmetry is broken down to $Q_{1}+Q_{2}+S=8+0+0$ [31], the enhanced supersymmetry gives rise to $2+2+2$ new supercharges including conformal ones. The total number of supercharges is the same as in the PPPP limit. We also derive the explicit form of these supercharges and their (anti)commutation relations to obtain the maximal super Schrödinger algebra. The last section is our conclusion.

\footnotetext{
${ }^{2}$ See the subsection 2.3 for the definitions of various nonrelativistic regimes.

${ }^{3}$ We denote the numbers of kinematical, dynamical, and conformal supercharges as $Q_{1}, Q_{2}$, and $S$ respectively.
} 


\section{Mass-deformed ABJM Theory}

The ABJM theory [4 was proposed as a candidate to describe multiple M2-brane systems. The model is a three-dimensional gauge theory which possesses $\mathcal{N}=6$ supersymmetry. The field content of this model is given by four bi-fundamental complex scalars $Y^{A}, A=1, \ldots, 4$, four bi-fundamental complex spinors $\Psi_{A}$ as well as two Chern-Simons gauge fields $A_{\mu}$ and $\hat{A}_{\mu}, \mu=$ $0,1,2$. The scalars and the fermions belong to the four dimensional complex representations of $R$-symmetry group $\mathrm{SU}(4)$. The $\mathrm{SU}(4)$ indices can be conveniently written in terms of more handy $\mathrm{SU}(2)_{\mathrm{L}} \times \mathrm{SU}(2)_{\mathrm{R}}$ notations: $A=\left(n, n^{\prime}\right)$, where $n$ and $n^{\prime}$ are $\mathrm{SU}(2)_{\mathrm{L}}$ and $\mathrm{SU}(2)_{\mathrm{R}}$ indices respectively. This decomposition is even more natural in the mass-deformed ABJM theory which has $\mathrm{SU}(2)_{\mathrm{L}} \times \mathrm{SU}(2)_{\mathrm{R}} \times \mathrm{U}(1)_{R}$ symmetry. Restriction to the sector of a single $\mathrm{SU}(2)$ factor gives the minimal model [6]. For $\mathrm{SU}(2)$ indices we will use the Latin $m$ and $n$ for the $\mathrm{SU}(2)_{\mathrm{L}}$ as well as primed letters for the $\mathrm{SU}(2)_{\mathrm{R}}$.

As about spinor notations, we choose $(2+1)$-dimensional gamma matrices which satisfy $\gamma^{\mu} \gamma^{\nu}=$ $\eta^{\mu \nu}+\epsilon^{\mu \nu \rho} \gamma_{\rho}$ as $\gamma^{0}=i \sigma^{2}, \gamma^{1}=\sigma^{1}$, and $\gamma^{2}=\sigma^{3}$. The suppressed spinor indices are expressed by $\xi \chi \equiv \xi^{\alpha} \chi_{\alpha}$ and $\xi \gamma^{\mu} \chi=\xi^{\alpha} \gamma_{\alpha}^{\mu \beta} \chi_{\beta}$ for the two component spinors $\xi$ and $\chi$. For the gauge indices we use the convention of the Ref. [45].

\subsection{Mass deformation}

The SUSY preserving mass deformation was first constructed for the BLG theory [46, 47]. Then it was found that the ABJM theory also admits the SUSY preserving mass deformation [43, 44]. There are several methods to obtain the mass-deformed ABJM theory, such as $\mathcal{N}=1$ superfield formalism [47], $D$-term and $F$-term deformations [44] in $\mathcal{N}=2$ superfield formalism [45]. These different versions of mass-deformed ABJM theory are equivalent [48] and the M-theory origin of the mass-deformation was investigated in Refs. [49, 50]. Below we consider the mass deformed version of ABJM theory in more details.

The mass deformed ABJM theory with $\mathrm{U}(N) \times \mathrm{U}(N)$ gauge group is given by the action [43,44],

$$
S=c \int d t d^{2} x\left(\mathcal{L}_{0}+\mathcal{L}_{\mathrm{CS}}-V_{\text {ferm }}-V_{\text {bos }}-V_{\mathrm{m}}\right),
$$


where

$$
\begin{aligned}
\mathcal{L}_{0}= & \operatorname{tr}\left(\frac{1}{c^{2}} D_{t} Y_{A}^{\dagger} D_{t} Y^{A}-D_{i} Y_{A}^{\dagger} D_{i} Y^{A}+\frac{i}{c} \Psi^{\dagger A} \gamma^{0} D_{t} \Psi_{A}+i \Psi^{\dagger A} \gamma^{i} D_{i} \Psi_{A}\right) \\
\mathcal{L}_{\mathrm{CS}}= & \frac{k \hbar}{4 \pi} \epsilon^{\mu \nu \rho} \operatorname{tr}\left(A_{\mu} \partial_{\nu} A_{\rho}+\frac{2 i}{3} A_{\mu} A_{\nu} A_{\rho}-\hat{A}_{\mu} \partial_{\nu} \hat{A}_{\rho}-\frac{2 i}{3} \hat{A}_{\mu} \hat{A}_{\nu} \hat{A}_{\rho}\right) \\
V_{\mathrm{ferm}}= & \frac{2 \pi i}{k \hbar c} \operatorname{tr}\left(Y_{A}^{\dagger} Y^{A} \Psi^{\dagger B} \Psi_{B}-Y^{A} Y_{A}^{\dagger} \Psi_{B} \Psi^{\dagger B}+2 Y^{A} Y_{B}^{\dagger} \Psi_{A} \Psi^{\dagger B}-2 Y_{A}^{\dagger} Y^{B} \Psi^{\dagger A} \Psi_{B}\right. \\
& \left.\quad+\epsilon^{A B C D} Y_{A}^{\dagger} \Psi_{B} Y_{C}^{\dagger} \Psi_{D}-\epsilon_{A B C D} Y^{A} \Psi^{\dagger B} Y^{C} \Psi^{\dagger D}\right), \\
V_{\mathrm{bos}}= & -\frac{4 \pi^{2}}{3 k^{2} \hbar^{2} c^{2}} \operatorname{tr}\left(Y_{A}^{\dagger} Y^{A} Y_{B}^{\dagger} Y^{B} Y_{C}^{\dagger} Y^{C}+Y^{A} Y_{A}^{\dagger} Y^{B} Y_{B}^{\dagger} Y^{C} Y_{C}^{\dagger}+4 Y_{A}^{\dagger} Y^{B} Y_{C}^{\dagger} Y^{A} Y_{B}^{\dagger} Y^{C}\right. \\
& \left.\quad-6 Y^{A} Y_{B}^{\dagger} Y^{B} Y_{A}^{\dagger} Y^{C} Y_{C}^{\dagger}\right), \\
V_{\mathrm{m}}= & \operatorname{tr}\left[\frac{i m c}{\hbar} M_{A}^{B} \Psi^{\dagger A} \Psi_{B}+\frac{4 \pi m}{k \hbar^{2}} M_{B}^{C}\left(Y^{A} Y_{A}^{\dagger} Y^{B} Y_{C}^{\dagger}-Y_{A}^{\dagger} Y^{A} Y_{C}^{\dagger} Y^{B}\right)+\frac{m^{2} c^{2}}{\hbar^{2}} Y_{A}^{\dagger} Y^{A}\right]
\end{aligned}
$$

and the explicit dependence on the speed of light $c$ and Planck constant $\hbar$ is given.

The last term (2.2e) in the action (2.1) represents the mass deformation with $m$ being the mass parameter and $M_{A}{ }^{B}=\operatorname{diag}(1,1,-1,-1)$. Without this term equation (2.1) represents the original massless ABJM action. The covariant derivatives are defined as

$$
\begin{aligned}
D_{t} Y^{A} & =\partial_{t} Y^{A}+i A_{t} Y^{A}-i Y^{A} \hat{A}_{t}, A_{t}=c A_{0}=\text { finite }, \\
D_{i} Y^{A} & =\partial_{i} Y^{A}+i A_{i} Y^{A}-i Y_{A}^{\dagger} \hat{A}_{i} .
\end{aligned}
$$

\section{$2.2 \mathcal{N}=6$ Supersymmetry}

The action (2.1) of the massless ABJM theory is invariant with respect to the following $\mathcal{N}=6$ supersymmetry transformations,

$$
\begin{aligned}
& \delta Y^{A}=\mathrm{i} \omega^{A B} \Psi_{B}, \quad \delta Y_{A}^{\dagger}=\mathrm{i} \Psi^{\dagger B} \omega_{A B}, \\
& \delta \Psi_{A}=\gamma^{\mu} \omega_{A B} D_{\mu} Y^{B}+\frac{2 \pi}{k} \omega_{A B}\left(Y^{B} Y_{C}^{\dagger} Y^{C}-Y^{C} Y_{C}^{\dagger} Y^{B}\right)+\frac{4 \pi}{k} \omega_{B C} Y^{B} Y_{A}^{\dagger} Y^{C} \\
& \delta \Psi^{\dagger A}=-D_{\mu} Y_{B}^{\dagger} \omega^{A B} \gamma^{\mu}+\frac{2 \pi}{k} \omega^{A B}\left(Y_{C}^{\dagger} Y^{C} Y_{B}^{\dagger}-Y_{B}^{\dagger} Y^{C} Y_{C}^{\dagger}\right)-\frac{4 \pi}{k} \omega^{B C} Y_{B}^{\dagger} Y^{A} Y_{C}^{\dagger}, \\
& \delta A_{\mu}=-\frac{2 \pi}{k}\left(\omega^{A B} Y_{A}^{\dagger} \gamma_{\mu} \Psi_{B}+Y^{A} \Psi^{\dagger B} \gamma_{\mu} \omega_{A B}\right), \\
& \delta \hat{A}_{\mu}=-\frac{2 \pi}{k}\left(\omega^{A B} Y_{A}^{\dagger} \gamma_{\mu} \Psi_{B}+\Psi^{\dagger B} \gamma_{\mu} Y^{A} \omega_{A B}\right),
\end{aligned}
$$

where $\omega^{A B}=-\omega^{B A}=\left(\omega_{A B}\right)^{*}=\frac{1}{2} \epsilon^{A B C D} \omega_{C D}$. The supersymmetric parameters $\omega_{A B}$ and $\omega^{A B}$ are related to the $(2+1)$-dimensional six Majorana spinors $\varepsilon_{I}, I=1, \cdots, 6$, by

$$
\omega_{A B}=\varepsilon_{I}\left(\Gamma^{I}\right)_{A B}, \quad \omega^{A B}=\varepsilon_{I}\left(\Gamma^{I *}\right)^{A B} .
$$


As we mentioned above, the mass deformation preserves the full $\mathcal{N}=6$ supersymmetry, the only effect of such deformation being the modification of the spinor field transformation rules by the additional terms,

$$
\begin{aligned}
\delta_{m} \Psi_{A} & =m M_{A}{ }^{B} \omega_{B C} Y^{C}, \\
\delta_{m} \Psi^{\dagger A} & =m M_{B}^{A} \omega^{B C} Y_{C}^{\dagger} .
\end{aligned}
$$

\subsection{Nonrelativistic limit(s)}

Formally, nonrelativistic limit corresponds to the limit of large speed of light $c \rightarrow \infty$. However, this limit is not uniquely defined. Below we give a brief description of nonrelativistic limits of our interests in ABJM theory.

As was discussed before, the mass deformation breaks the original SU(4) $R$-symmetry down to $\mathrm{SU}(2)_{\mathrm{L}} \times \mathrm{SU}(2)_{\mathrm{R}} \times \mathrm{U}(1)_{R} R$-symmetry. According to this the fields are split as,

$$
\begin{array}{ll}
Y^{A}=\left(Y^{n}, Y^{n^{\prime}}\right), & Y_{A}^{\dagger}=\left(Y_{n}^{\dagger}, Y_{n^{\prime}}^{\dagger}\right), \\
\Psi_{A}=\left(\Psi_{n}, \Psi_{n^{\prime}}\right), & \Psi^{\dagger A}=\left(\Psi^{\dagger n}, \Psi^{\dagger n^{\prime}}\right),
\end{array}
$$

where $A=1,2,3,4, n=1,2$, and $n^{\prime}=3,4$.

In order to go to the nonrelativistic limit, we decompose the relativistic fields into the particle and antiparticle parts,

$$
Y^{n}=\frac{\hbar}{\sqrt{2 m}}\left(\mathrm{e}^{-\mathrm{i} m c^{2} t / \hbar} y^{n}+\mathrm{e}^{\mathrm{i} m c^{2} t / \hbar} \hat{y}^{\dagger n}\right), \quad \Psi_{n}=\sqrt{\hbar c}\left(\mathrm{e}^{-\mathrm{i} m c^{2} t / \hbar} \psi_{n}+\mathrm{e}^{\mathrm{i} m c^{2} t / \hbar} \sigma_{2} \hat{\psi}_{n}^{\dagger}\right),
$$

and analogously for $Y^{n^{\prime}}$ and $\Psi_{n^{\prime}}$. Here the minus sign in the exponent $\mathrm{e}^{ \pm \mathrm{i} m c^{2} t / \hbar}$ corresponds to a particle, while plus sign comes with an antiparticle.

In this situation we can make a choice for each of complex fields $\left(Y^{n}, Y^{n^{\prime}}, \Psi_{n}, \Psi_{n^{\prime}}\right)$ to be either particle (P), or antiparticle (A) separately. Following [31], we will denote such a choice by a four letter string consisting of 'P's and 'A's, e.g. the limit with all fields chosen to be in purely particle sector is denoted PPPP; the limit in which $Y^{n}$ and $\Psi_{n^{\prime}}$ are particles while $Y^{n^{\prime}}$ and $\Psi_{n}$ antiparticles is denoted PAAP, etc.

To obtain the nonrelativistic limit we have to plug the Ansatz (2.8) (where the choice is made for the particles or antiparticles) into the mass-deformed action (2.1), take the limit $c \rightarrow \infty$, keeping the leading terms. In the case of fermions we have to eliminate the heavy modes using their equations of motion [31,32].

\section{PAAP Limit}

The basic supersymmetry in various nonrelativistic limits was studied in [31], where the number of supersymmetries was found for each limit labeled by the four-letter string. In general, the 
number of supercharges depends on the relative choice of fields as particles or antiparticles. The maximal supersymmetry is reached in the case of either PPPP, when all fields are particles or AAAA, when all are antiparticles. In this case the theory possesses 14 supercharges of which 10 supercharges are kinematical, 2 are dynamical, and the remaining 2 are conformal related to super Schrödinger symmetry. Other cases contain less supersymmetry of this kind [31]. That is, if we include antiparticle sectors in nonrelativistic limits of the ABJM theory, the number of supercharges is reduced. This is claimed to be a general property in the nonrelativistic limits of superconformal field theories [41,42]. For instance, the PAAP case, which we are going to consider in more details below, possesses only eight kinematical supercharges and no conformal supercharges are present, i.e. $Q_{1}+Q_{2}+S=8+0+0$.

As we know about the SUSY enhancement in relativistic ABJM theory, we have to use the monopole operators to obtain the additional $\mathcal{N}=2$ supersymmetry [5, 6]. By using monopole operators, we can include both 4 and $\overline{4}$ matter fields simultaneously in $\mathcal{N}=8$ supersymmetry transformation rules for $k=1,2$. In the nonrelativistic limit, however, we cannot do this, since we have to choose between particle or antiparticle parts for a given relativistic field. (See the subsection 4.1 for the detailed explanations.) Therefore the $\mathcal{N}=8$ supersymmetry in relativistic ABJM theory for $k=1,2$ is inevitably broken in any nonrelativistic limit.

So far, the most supersymmetric case is the PPPP limit with 14 supercharges. However, since the monopole operators generate supersymmetry transformations which mix the 4 and $\overline{4}$ representations, we can expect other limits with maximal supersymmetry, which will include antiparticle sectors. Indeed, we find that the PAAP limit is another case which has 14 supercharges using monopole operators.

\subsection{Action}

Let us consider the PAAP case in more details. In PAAP limit one chooses the first boson and second fermion to be in particle sector while second boson and first fermion to be antiparticles,

$$
\begin{aligned}
Y^{n} & =\frac{\hbar}{\sqrt{2 m}} e^{-i m c^{2} t / \hbar} y^{n}, & Y_{n}^{\dagger} & =\frac{\hbar}{\sqrt{2 m}} e^{i m c^{2} t / \hbar} y_{n}^{\dagger}, \\
Y^{n^{\prime}} & =\frac{\hbar}{\sqrt{2 m}} e^{i m c^{2} t / \hbar} \hat{y}^{\dagger n^{\prime}}, & Y_{n^{\prime}}^{\dagger} & =\frac{\hbar}{\sqrt{2 m}} e^{-i m c^{2} t / \hbar} \hat{y}_{n^{\prime}}, \\
\Psi_{n} & =\sqrt{\hbar c} e^{i m c^{2} t / \hbar} \sigma_{2} \hat{\psi}_{n}^{\dagger}, & \Psi^{\dagger n} & =-\sqrt{\hbar c} e^{-i m c^{2} t / \hbar} \sigma_{2} \hat{\psi}^{n}, \\
\Psi_{n^{\prime}} & =\sqrt{\hbar c} e^{-i m c^{2} t / \hbar} \psi_{n^{\prime}}, & \Psi^{\dagger n^{\prime}} & =\sqrt{\hbar c} e^{i m c^{2} t / \hbar} \psi^{\dagger n^{\prime}} .
\end{aligned}
$$

Applying the general procedure for nonrelativistic limit mentioned before, we obtain the following nonrelativistic action,

$$
S_{\text {PAAP }}=\int \mathrm{d} t \mathrm{~d}^{2} x\left(\mathcal{L}_{\text {scalar }}+\mathcal{L}_{\text {fermion }}+\mathcal{L}_{C S}\right)
$$


where the scalar and fermionic parts of the Lagrangian $\mathcal{L}_{\text {scalar }}$ and $\mathcal{L}_{\text {fermion }}$ are given, respectively, by:

$$
\begin{aligned}
\mathcal{L}_{\text {scalar }}=\operatorname{tr}[ & i \hbar y_{n}^{\dagger} D_{t} y^{n}+i \hbar \hat{y}^{\dagger n^{\prime}} D_{t} \hat{y}_{n^{\prime}}-\frac{\hbar^{2}}{2 m} D_{i} y_{n}^{\dagger} D_{i} y^{n}-\frac{\hbar^{2}}{2 m} D_{i} \hat{y}_{n^{\prime}} D_{i} \hat{y}^{\dagger n^{\prime}} \\
& \left.-\frac{\pi \hbar^{2}}{k m}\left(y^{n} y_{n}^{\dagger} y^{m} y_{m}^{\dagger}-\hat{y}^{\dagger n^{\prime}} \hat{y}_{n^{\prime}} \hat{y}^{\dagger m^{\prime}} \hat{y}_{m^{\prime}}-y_{n}^{\dagger} y^{n} y_{m}^{\dagger} y^{m}+\hat{y}_{n^{\prime}} \hat{y}^{\dagger n^{\prime}} \hat{y}_{m^{\prime}} \hat{y}^{\dagger m^{\prime}}\right)\right]
\end{aligned}
$$

and

$$
\begin{aligned}
& \mathcal{L}_{\text {fermion }}= i \hbar \operatorname{tr}\left(\hat{\psi}_{+n} D_{t} \hat{\psi}_{+}^{n}+\psi_{-}^{\dagger n^{\prime}} D_{t} \psi_{-n^{\prime}}\right)-\frac{\hbar^{2}}{2 m} \operatorname{tr}\left(D_{i} \hat{\psi}_{+n}^{\dagger} D_{i} \hat{\psi}_{+}^{n}+D_{i} \psi_{-}^{\dagger n^{\prime}} D_{i} \psi_{-n^{\prime}}\right) \\
&+\frac{\hbar^{2}}{2 m} \operatorname{tr}\left(\hat{\psi}_{+n}^{\dagger} \hat{F}_{12} \hat{\psi}_{+}^{n}-F_{12} \hat{\psi}_{+n}^{\dagger} \hat{\psi}_{+}^{n}-\psi_{-}^{\dagger n^{\prime}} F_{12} \psi_{-n^{\prime}}+\hat{F}_{12} \psi_{-}^{\dagger n^{\prime}} \psi_{-n^{\prime}}\right) \\
&+\frac{\pi \hbar^{2}}{k m} \operatorname{tr}\left[\left(y_{n}^{\dagger} y^{n}+\hat{y}_{n^{\prime}} \hat{y}^{\dagger n^{\prime}}\right)\left(\hat{\psi}_{+}^{m} \hat{\psi}_{+m}^{\dagger}+\psi_{-}^{\dagger m^{\prime}} \psi_{-m^{\prime}}\right)+\left(y^{n} y_{n}^{\dagger}+\hat{y}^{\dagger n^{\prime}} \hat{y}_{n^{\prime}}\right)\left(\hat{\psi}_{+m}^{\dagger} \hat{\psi}_{+}^{m}+\psi_{-m^{\prime}} \psi_{-}^{\dagger m^{\prime}}\right)\right. \\
& \quad-2\left(y^{n} y_{m}^{\dagger} \hat{\psi}_{+n}^{\dagger} \hat{\psi}_{+}^{m}-y^{n} \hat{y}_{m^{\prime}} \hat{\psi}_{+n}^{\dagger} \psi_{-}^{\dagger m^{\prime}}-\hat{y}^{\dagger n^{\prime}} y_{m}^{\dagger} \psi_{-n^{\prime}} \hat{\psi}_{+}^{m}+\hat{y}^{\dagger n^{\prime}} \hat{y}_{m^{\prime}} \psi_{-n^{\prime}} \psi_{-}^{\dagger m^{\prime}}\right) \\
&\left.\quad-2\left(y_{n}^{\dagger} y^{m} \hat{\psi}_{+}^{n} \hat{\psi}_{+m}^{\dagger}-y_{m}^{\dagger} \hat{y}^{\dagger m^{\prime}} \hat{\psi}_{+}^{n} \psi_{-m^{\prime}}-\hat{y}_{n^{\prime}} y^{m} \psi_{-}^{\dagger n^{\prime}} \hat{\psi}_{+m}^{\dagger}+\hat{y}_{n^{\prime}} \hat{y}^{\dagger m^{\prime}} \psi_{-}^{\dagger n^{\prime}} \psi_{-m^{\prime}}\right)\right], \quad(3.4)
\end{aligned}
$$

while the Chern-Simons part $\mathcal{L}_{C S}$ is still given by $(2.2 \mathrm{~b})$.

In deriving the fermionic part of the Lagrangian (3.4), we used the equations of motion for $\hat{\psi}_{-n}^{\dagger}$ and $\psi_{+}^{\dagger n^{\prime}}$

$$
\begin{aligned}
\hat{\psi}_{-}^{n} & =-\frac{i \hbar}{2 m c} D_{-} \hat{\psi}_{+}^{n}-\frac{i \hbar}{2 m c^{2}} D_{t} \hat{\psi}_{-}^{n}=-\frac{i \hbar}{2 m c} D_{-} \hat{\psi}_{+}^{n}+\mathcal{O}\left(\frac{1}{m^{2} c^{3}}\right), \\
\psi_{+n^{\prime}} & =\frac{i \hbar}{2 m c} D_{+} \psi_{-n^{\prime}}-\frac{i \hbar}{2 m c^{2}} D_{t} \psi_{+n^{\prime}}=\frac{i \hbar}{2 m c} D_{+} \psi_{-n^{\prime}}+\mathcal{O}\left(\frac{1}{m^{2} c^{3}}\right),
\end{aligned}
$$

to eliminate the 'heavy modes' $\hat{\psi}_{-}^{n}$ and $\psi_{+n^{\prime}}$, and $D_{ \pm}=D_{1} \pm i D_{2}$.

\subsection{8 kinematical supercharges}

The action (3.2) for nonrelativistic PAAP limit is invariant with respect to supersymmetry transformations. In Ref. [31, eight supersymmetry transformations were found. This supersymmetry is all kinematical and given by

$$
\begin{array}{ll}
\delta y^{n}=-\eta_{+}^{n n^{\prime}} \psi_{-n^{\prime}}, & \delta y_{n}^{\dagger}=\eta_{-n n^{\prime}} \psi_{-}^{\dagger n^{\prime}}, \\
\delta \hat{y}_{n^{\prime}}=\eta_{-n n^{\prime}} \hat{\psi}_{+}^{n}, & \delta \hat{y}^{\dagger n^{\prime}}=-\eta_{+}^{n n^{\prime}} \hat{\psi}_{+n}^{\dagger}, \\
\delta \hat{\psi}_{+}^{n}=-\eta_{+}^{n n^{\prime}} \hat{y}_{n^{\prime}}, & \delta \hat{\psi}_{+n}^{\dagger}=-\eta_{-n n^{\prime}} \hat{y}^{\dagger n^{\prime}}, \\
\delta \psi_{-n^{\prime}}=\eta_{-n n^{\prime}} y^{n}, & \delta \psi_{-}^{\dagger n^{\prime}}=\eta_{+}^{n n^{\prime}} y_{n}^{\dagger}, \\
\delta A_{t}=-\frac{\pi \hbar}{k m}\left(\hat{y}^{\dagger n^{\prime}} \eta_{-n n^{\prime}} \hat{\psi}_{+}^{n}+\hat{\psi}_{+n}^{\dagger} \eta_{+}^{n n^{\prime}} \hat{y}_{n^{\prime}}-\eta_{+}^{n n^{\prime}} \psi_{-n^{\prime}} y_{n}^{\dagger}-y^{n} \psi_{-}^{\dagger n^{\prime}} \eta_{-n n^{\prime}}\right), \\
\delta \hat{A}_{t}=-\frac{\pi \hbar}{k m}\left(\eta_{-n n^{\prime}} \hat{\psi}_{+}^{n} \hat{y}^{\dagger n^{\prime}}+\hat{y}_{n^{\prime}} \hat{\psi}_{+n}^{\dagger} \eta_{+}^{n n^{\prime}}-y_{n}^{\dagger} \eta_{+}^{n n^{\prime}} \psi_{-n^{\prime}}-\psi_{-}^{\dagger n^{\prime}} \eta_{-n n^{\prime}} y^{n}\right), \\
\delta A_{ \pm}=\delta \hat{A}_{ \pm}=0,
\end{array}
$$


where $A_{ \pm} \equiv A_{1} \pm i A_{2}$ and $\hat{A}_{ \pm} \equiv \hat{A}_{1} \pm i \hat{A}_{2}$ and the one-component spinor parameters $\eta_{ \pm}$satisfy the following conditions,

$$
\eta_{ \pm n n^{\prime}}=\frac{1}{2} \epsilon_{n m} \epsilon_{n^{\prime} m^{\prime}} \eta_{ \pm}^{m m^{\prime}}, \quad \eta_{+}^{n n^{\prime}}=-\eta_{+}^{n^{\prime} n}, \quad \eta_{-n n^{\prime}}=-\eta_{-n^{\prime} n}
$$

with $\epsilon_{12}=\epsilon_{3^{\prime} 4^{\prime}}=1$.

\subsection{Superalgebra}

Let us consider the superalgebra of symmetries of the action (3.2). In order to do this we rewrite the action (3.2) in a form more appropriate for canonical analysis,

$$
\begin{aligned}
\mathcal{L}_{\mathrm{PAAP}}= & i \hbar \operatorname{tr}\left(y_{n}^{\dagger} \partial_{t} y^{n}+\hat{y}^{\dagger n^{\prime}} \partial_{t} \hat{y}_{n^{\prime}}+\hat{\psi}_{+n}^{\dagger} \partial_{t} \hat{\psi}_{+}^{n}+\psi_{-}^{\dagger n^{\prime}} \partial_{t} \psi_{-n^{\prime}}\right)-\hbar^{2} \mathcal{H} \\
& +\frac{k \hbar}{2 \pi} \operatorname{tr}\left(A_{2} \partial_{t} A_{1}-\hat{A}_{2} \partial_{t} \hat{A}_{1}+A_{t} \mathcal{G}-\hat{A}_{t} \hat{\mathcal{G}}\right)
\end{aligned}
$$

where the Hamiltonian density is given by

$$
\begin{aligned}
\mathcal{H}=\frac{1}{2 m} \operatorname{tr} & D_{i} y_{n}^{\dagger} D_{i} y^{n}+D_{i} \hat{y}^{\dagger n^{\prime}} D_{i} \hat{y}_{n^{\prime}}+D_{i} \hat{\psi}_{+n}^{\dagger} D_{i} \hat{\psi}_{+}^{n}+D_{i} \psi_{-}^{\dagger n^{\prime}} D_{i} \psi_{-n^{\prime}} \\
& \left.-\hat{\psi}_{+n}^{\dagger} \hat{F}_{12} \hat{\psi}_{+}^{n}+F_{12} \hat{\psi}_{+n}^{\dagger} \hat{\psi}_{+}^{n}+\psi_{-}^{\dagger n^{\prime}} F_{12} \psi_{-n^{\prime}}-\hat{F}_{12} \psi_{-}^{\dagger n^{\prime}} \psi_{-n^{\prime}}\right) \\
+\frac{\pi}{k m} \operatorname{tr}[ & y^{n} y_{n}^{\dagger} y^{m} y_{m}^{\dagger}-\hat{y}^{\dagger n^{\prime}} \hat{y}_{n^{\prime}} \hat{y}^{\dagger m^{\prime}} \hat{y}_{m^{\prime}}-y_{n}^{\dagger} y^{n} y_{m}^{\dagger} y^{m}+\hat{y}_{n^{\prime}} \hat{y}^{\dagger n^{\prime}} \hat{y}_{m^{\prime}} \hat{y}^{\dagger m^{\prime}} \\
& -\left(y_{n}^{\dagger} y^{n}+\hat{y}_{n^{\prime}} \hat{y}^{\dagger n^{\prime}}\right)\left(\hat{\psi}_{+}^{m} \hat{\psi}_{+m}^{\dagger}+\psi_{-}^{\dagger m^{\prime}} \psi_{-m^{\prime}}\right)-\left(y^{n} y_{n}^{\dagger}+\hat{y}^{\dagger n^{\prime}} \hat{y}_{n^{\prime}}\right)\left(\hat{\psi}_{+m}^{\dagger} \hat{\psi}_{+}^{m}+\psi_{-m^{\prime}} \psi_{-}^{\dagger m^{\prime}}\right) \\
& +2\left(y^{n} y_{m}^{\dagger} \hat{\psi}_{+n}^{\dagger} \hat{\psi}_{+}^{m}-y^{n} \hat{y}_{m^{\prime}} \hat{\psi}_{+n}^{\dagger} \psi_{-}^{\dagger m^{\prime}}-\hat{y}^{\dagger n^{\prime}} y_{m}^{\dagger} \psi_{-n^{\prime}} \hat{\psi}_{+}^{m}+\hat{y}^{\dagger n^{\prime}} \hat{y}_{m^{\prime}} \psi_{-n^{\prime}} \psi_{-}^{\dagger m^{\prime}}\right) \\
& \left.+2\left(y_{n}^{\dagger} y^{m} \hat{\psi}_{+}^{n} \hat{\psi}_{+m}^{\dagger}-y_{m}^{\dagger} \hat{y}^{\dagger m^{\prime}} \hat{\psi}_{+}^{n} \psi_{-m^{\prime}}-\hat{y}_{n^{\prime}} y^{m} \psi_{-}^{\dagger n^{\prime}} \hat{\psi}_{+m}^{\dagger}+\hat{y}_{n^{\prime}} \hat{y}^{\dagger m^{\prime}} \psi_{-}^{\dagger n^{\prime}} \psi_{-m^{\prime}}\right)\right],
\end{aligned}
$$

and the Gauss law constraints are

$$
\begin{aligned}
& \mathcal{G}=F_{12}-\frac{2 \pi}{k}\left(y^{n} y_{n}^{\dagger}-\hat{y}^{\dagger n^{\prime}} \hat{y}_{n^{\prime}}-\hat{\psi}_{+n}^{\dagger} \hat{\psi}_{+}^{n}-\psi_{-n^{\prime}} \psi_{-}^{\dagger n^{\prime}}\right), \\
& \hat{\mathcal{G}}=\hat{F}_{12}-\frac{2 \pi}{k}\left(y_{n}^{\dagger} y^{n}-\hat{y}_{n^{\prime}} \hat{y}^{\dagger n^{\prime}}+\hat{\psi}_{+}^{n} \hat{\psi}_{+n}^{\dagger}+\psi_{-}^{\dagger n^{\prime}} \psi_{-n^{\prime}}\right) .
\end{aligned}
$$

In the following subsections, we will first derive the superalgebra associated with the supersymmetry transformation given in (3.6). The analysis parallels to that of the PPPP case [31]. The supersymmetry coming from the monopole operator will be studied in the next section. The canonical commutation relations are given from (3.8):

$$
\begin{aligned}
& {\left[A_{+}(x)_{b}^{a}, A_{-}(y)_{d}^{c}\right]=\frac{4 \pi}{k} \delta_{d}^{a} \delta_{b}^{c} \delta^{2}(x-y), \quad\left[\hat{A}_{+}(x)_{\hat{b}}^{\hat{a}}, \hat{A}_{-}(y)_{\hat{d}}^{\hat{c}}\right]=-\frac{4 \pi}{k} \delta_{\hat{d}}^{\hat{a}} \delta_{\hat{b}}^{\hat{c}} \delta^{2}(x-y),} \\
& {\left[y^{n}(x)_{\hat{a}}^{a}, y_{m}^{\dagger}(y)_{b}^{\hat{b}}\right]=\delta_{m}^{n} \delta_{b}^{a} \delta_{\hat{a}}^{\hat{b}} \delta^{2}(x-y), \quad\left[\hat{y}_{n^{\prime}}(x)_{a}^{\hat{a}}, \hat{y}^{\dagger m^{\prime}}(y)_{\hat{b}}^{b}\right]=\delta_{n^{\prime}}^{m^{\prime}} \delta_{a}^{b} \delta_{\hat{b}}^{\hat{a}} \delta^{2}(x-y),} \\
& \left\{\hat{\psi}_{+}^{n}(x)_{a}^{\hat{a}}, \hat{\psi}_{+m}^{\dagger}(y)_{\hat{b}}^{b}\right\}=\delta_{m}^{n} \delta_{a}^{b} \delta_{\hat{b}}^{\hat{a}} \delta^{2}(x-y), \quad\left\{\psi_{-n^{\prime}}(x)_{\hat{a}}^{a}, \psi_{-}^{\dagger m^{\prime}}(y)_{b}^{\hat{b}}\right\}=\delta_{n^{\prime}}^{m^{\prime}} \delta_{b}^{a} \delta_{\hat{a}}^{\hat{b}} \delta^{2}(x-y) .
\end{aligned}
$$




\subsubsection{Bosonic Schrödinger algebra}

Let us first consider the bosonic subalgebra. Applying Noether techniques we find that the bosonic symmetry algebra consists of the following conserved charges:

- Hamiltonian

$$
H=\int d^{2} x \mathcal{H}
$$

- Linear momentum

$$
P_{i}=\int d^{2} x \mathcal{P}_{i}
$$

where the momentum density is given by

$$
\begin{aligned}
\mathcal{P}_{i}=-\frac{i}{2} \operatorname{tr} & \left(y_{n}^{\dagger} D_{i} y^{n}-D_{i} y_{n}^{\dagger} y^{n}+\hat{y}^{\dagger n^{\prime}} D_{i} \hat{y}_{n^{\prime}}-D_{i} \hat{y}^{\dagger n^{\prime}} \hat{y}_{n^{\prime}}\right. \\
& \left.+\hat{\psi}_{+n}^{\dagger} D_{i} \hat{\psi}_{+}^{n}-D_{i} \hat{\psi}_{+n}^{\dagger} \hat{\psi}_{+}^{n}+\psi_{-}^{\dagger n^{\prime}} D_{i} \psi_{-n^{\prime}}-D_{i} \psi_{-}^{\dagger n^{\prime}} \psi_{-n^{\prime}}\right) .
\end{aligned}
$$

- Angular momentum

$$
J=\int d^{2} x \epsilon_{i j} x_{i} \mathcal{P}_{j}+\Sigma
$$

where the spin $\Sigma$ is given by

$$
\Sigma=\frac{1}{2} \int d^{2} x \operatorname{tr}\left(\psi_{-}^{\dagger n^{\prime}} \psi_{-n^{\prime}}-\hat{\psi}_{+n}^{\dagger} \hat{\psi}_{+}^{n}\right)
$$

- Total number operator

$$
\mathcal{N}=\int d^{2} x n(x)
$$

with number density given by

$$
n(x)=\operatorname{tr}\left(y_{n}^{\dagger} y^{n}+\hat{y}^{\dagger n^{\prime}} \hat{y}_{n^{\prime}}+\hat{\psi}_{+n}^{\dagger} \hat{\psi}_{+}^{n}+\psi_{-}^{\dagger n^{\prime}} \psi_{-n^{\prime}}\right) .
$$

- Galilean boost

$$
G_{i}=-t P_{i}+m \int d^{2} x x_{i} n(x)
$$

- Dilatation

$$
D=2 t H-\int d^{2} x x_{i} \mathcal{P}_{i}
$$


- Special conformal transformation

$$
K=-t^{2} H+t D+\frac{m}{2} \int d^{2} x x^{2} n(x) .
$$

These operators are the subjects to the following algebraic relations defining the bosonic Schrödinger algebra [51]:

$$
\begin{aligned}
& i[D, H]=2 H, \quad i[D, K]=-2 K, \quad i[K, H]=D, \\
& i\left[H, G_{i}\right]=P_{i}, \quad i\left[K, P_{i}\right]=-G_{i}, \quad i\left[P_{i}, G_{i}\right]=\delta_{i j} m \mathcal{N}, \\
& i\left[D, P_{i}\right]=P_{i}, \quad i\left[D, G_{i}\right]=-G_{i}, \quad i\left[J, P_{i}\right]=-\epsilon_{i j} P_{j}, \quad i\left[J, G_{i}\right]=-\epsilon_{i j} G_{j} .
\end{aligned}
$$

In the above algebra $H, D, K$ are the generators of the conformal subgroup $S O(2,1)$ and the number operator $\mathcal{N}$ appears as a central term.

\subsubsection{Kinematical superalgebra}

The bosonic algebra can be enlarged to superalgebra by adding the 8 kinematic fermionic charges generating the transformations (3.6),

$$
\begin{gathered}
q_{+}^{n n^{\prime}}=i \int d^{2} x \operatorname{tr}\left(\psi_{-}^{\dagger n^{\prime}} y^{n}-\hat{y}^{\dagger n^{\prime}} \hat{\psi}_{+}^{n}\right), \\
q_{-n n^{\prime}}=i \int d^{2} x \operatorname{tr}\left(\hat{\psi}_{+n}^{\dagger} \hat{y}_{n^{\prime}}-y_{n}^{\dagger} \psi_{-n^{\prime}}\right),
\end{gathered}
$$

as well as $S U(2)_{\mathrm{L}}$ and $S U(2)_{\mathrm{R}} R$-symmetry generators

$$
\begin{aligned}
& R_{m}^{n}=-\int d^{2} x \operatorname{tr}\left[y_{m}^{\dagger} y^{n}+\hat{\psi}_{+m}^{\dagger} \hat{\psi}_{+}^{n}-\frac{1}{2} \delta_{m}^{n}\left(y_{l}^{\dagger} y^{l}+\hat{\psi}_{+l}^{\dagger} \hat{\psi}_{+}^{l}\right)\right], \\
& R_{m^{\prime}}^{n^{\prime}}=\int d^{2} x \operatorname{tr}\left[\hat{y}^{\dagger n^{\prime}} \hat{y}_{m^{\prime}}+\psi_{-}^{\dagger n^{\prime}} \psi_{-m^{\prime}}-\frac{1}{2} \delta_{m^{\prime}}^{n^{\prime}}\left(\hat{y}^{\dagger l^{\prime}} \hat{y}_{l^{\prime}}+\psi_{-}^{\dagger l^{\prime}} \psi_{-l^{\prime}}\right)\right] .
\end{aligned}
$$

They rotate the fields and the generators with $S U(2)_{\mathrm{L}}$ and $S U(2)_{\mathrm{R}}$ indices. Therefore they satisfy the standard $\mathrm{su}(2)$ algebra. The supercharges satisfy the following commutation relations

$$
\begin{gathered}
\left\{q_{+}^{n n^{\prime}}, q_{-m m^{\prime}}\right\}=\frac{1}{2} \delta_{m^{\prime}}^{n^{\prime}} \delta_{m}^{n} \mathcal{N}+\delta_{m}^{n} R_{m^{\prime}}^{n^{\prime}}-\delta_{m^{\prime}}^{n^{\prime}} R_{m}^{n}, \\
{\left[J, q_{+}^{n n^{\prime}}\right]=\frac{1}{2} q_{+}^{n n^{\prime}}, \quad\left[J, q_{-n n^{\prime}}\right]=-\frac{1}{2} q_{-n n^{\prime}}}
\end{gathered}
$$

Along with Schrödinger algebra (3.22), the commutation relations (3.25) describe a kinematical superalgebra.

In the case of PPPP, there exists an $U(1)_{R}$ symmetry in the non-relativistic limit coming from the reduction of $S U(4) R$-symmetry to $S U(2)_{\mathrm{L}} \times S U(2)_{\mathrm{R}} \times U(1)_{R}$ symmetry. We will see that in the PAAP case also, there is a similar $U(1)_{R}$ symmetry connecting the particle and antiparticle sectors. 


\section{SUSY Enhancement with Monopole Operators in Non- relativistic Limit}

In this section we find the additional supercharges and the corresponding superalgebra in the PAAP limit. We emphasize the fundamental role of monopole operators in the supersymmetry enhancement for both relativistic and nonrelativistic superconformal field theories.

\subsection{SUSY enhancement and monopole operators}

For the Chern-Simons level $k=1,2$ the ABJM theory was shown to be a subject to the additional hidden $\mathcal{N}=2$ supersymmetry [5, 6]. The additional supersymmetry transformations involve the monopole operators. In this subsection we investigate the role of monopole operators in supersymmetry enhancement of (mass deformed) ABJM theory and its nonrelativistic limit.

The ABJM theory described by the action (2.1) contains complex representations for matter fields and it has $\mathrm{SU}(4) \times \mathrm{U}(1)$ global symmetry corresponding to the transverse space to M2branes. To find the hidden symmetry in the transverse space we should use both the $\mathbf{4}$ and $\overline{4}$-complex representations simultaneously. However, in order to include both of them in the same supersymmetry transformation, we have to modify one of the representations by the help of a monopole operator.

Attaching the monopole operator to a matter field changes the gauge transformation rule of the field without changing the global symmetry properties. For instance, a bi-fundamental scalar field $Y^{A}$ in 4-representation can be converted to an anti-bi-fundamental one $\tilde{Y}^{A}$ in the same 4-complex representation as follows,

$$
\tilde{Y}^{A \hat{a}}=\bar{\tau}_{a b}^{\hat{a} \hat{b}} Y_{\hat{b}}^{A b}
$$

where $\bar{\tau}_{a b}^{\hat{a} \hat{b}}$ is the monopole operator related to the supersymmetry enhancement. For the $\mathrm{U}(2) \times \mathrm{U}(2)$ case, the explicit form of the monopole operator is given by [6]

$$
\bar{\tau}_{a b}^{\hat{a} \hat{b}}=e^{-2 i \int_{x}^{\infty} A_{\mu}^{-} d x^{\mu}} \epsilon^{\hat{a} \hat{b}} \epsilon_{a b}
$$

where $\epsilon_{a b}$ and $\epsilon^{\hat{a} \hat{b}}$ are invariant antisymmetric tensors for left and right $\mathrm{SU}(2)$ parts of the gauge group respectively.

Attaching a monopole operator to a local field also does not change the local nature of the resulting composite field for $k=1,2$. In this case, we can introduce in supersymmetry transformations new local fields, which do not exist in the original ABJM theory. This fact was demonstrated for the abelian ABJM theory [5].

Therefore the enhanced supersymmetry transformation rule takes the following form [6]

$$
\delta Y^{A}=i \omega^{A B} \Psi_{B}+i \tilde{\varepsilon} \tilde{\Psi}^{\dagger A}
$$


where $\tilde{\Psi}_{\hat{a}}^{\dagger A a}=\tau_{\hat{a} \hat{b}}^{a b} \Psi_{b}^{\dagger A \hat{b}}$ is in the bi-fundamental representation and $\tilde{\varepsilon}$ is a complex spinor. As we see in (4.3), we have to use the 4 and $\overline{4}$-representations simultaneously in order to obtain the additional $\mathcal{N}=2$ supersymmetry. Due to the presence of the monopole operator both sides of expression in (4.3) can be in the bi-fundamental representations of the gauge group.

Now let us consider the supersymmetric properties and the role of monopole operator in nonrelativistic limits. Even though it is possible to obtain the supersymmetry transformation rules in nonrelativistic limit by reduction from those of relativistic theory, we use different strategy. Instead we analyze full supersymmetry of the nonrelativistic action. However, taking into account the nature of the monopole operators, we can roughly estimate the supersymmetric properties for a given choice of particle and antiparticle sectors.

In PPPP limit, (4.3) is reduced to

$$
\begin{aligned}
& \delta y^{n}=i \sqrt{\frac{2 m c}{\hbar}} \omega^{n m} \psi_{m}+i \sqrt{\frac{2 m c}{\hbar}} \omega^{n m^{\prime}} \psi_{m^{\prime}}+i \sqrt{\frac{2 m c}{\hbar}} e^{2 i m c^{2} t / \hbar} \tilde{\varepsilon} \tilde{\psi}^{\dagger n} \\
& \delta y^{n^{\prime}}=i \sqrt{\frac{2 m c}{\hbar}} \omega^{n^{\prime} m} \psi_{m}+i \sqrt{\frac{2 m c}{\hbar}} \omega^{n^{\prime} m^{\prime}} \psi_{m^{\prime}}+i \sqrt{\frac{2 m c}{\hbar}} e^{2 i m c^{2} t / \hbar} \tilde{\varepsilon} \tilde{\psi}^{\dagger n^{\prime}}
\end{aligned}
$$

where $\tilde{\psi}_{\hat{a}}^{\dagger A a}=\tau_{\hat{a} \hat{b}}^{a b} \psi_{b}^{\dagger A \hat{b}}$. Dropping the fast oscillating terms in the nonrelativistic limit $c \rightarrow \infty$ in (4.4), we see that the supersymmetry parameter $\tilde{\varepsilon}$ in (4.4) does not exist in the limit. This means that the additional $\mathcal{N}=2$ supersymmetry with the monopole operator is broken in the PPPP limit. Then the PPPP limit has only 12 supercharges mentioned in Refs. [31, 32, which are inherited from the original $\mathcal{N}=6$ supersymmetry in the ABJM theory. There are also 2 conformal supercharges which are new in nonrelativistic limit.

On the other hand, for the PAAP limit for $k=1,2$, (4.3) becomes

$$
\begin{aligned}
\delta y^{n} & =i \sqrt{\frac{2 m c}{\hbar}} e^{2 i m c^{2} t / \hbar} \omega^{n m} \sigma_{2} \hat{\psi}_{m}^{\dagger}+i \sqrt{\frac{2 m c}{\hbar}} \omega^{n m^{\prime}} \psi_{m^{\prime}}+i \sqrt{\frac{2 m c}{\hbar}} \tilde{\varepsilon} \tilde{\hat{\psi}}^{n} \\
\delta \hat{y}^{\dagger n^{\prime}} & =i \sqrt{\frac{2 m c}{\hbar}} \omega^{n^{\prime} m} \sigma_{2} \hat{\psi}_{m}^{\dagger}+i \sqrt{\frac{2 m c}{\hbar}} e^{-2 i m c^{2} t / \hbar} \omega^{n^{\prime} m^{\prime}} \psi_{m^{\prime}}+i \sqrt{\frac{2 m c}{\hbar}} \tilde{\varepsilon} \tilde{\psi}^{\dagger n^{\prime}},
\end{aligned}
$$

where $\tilde{\hat{\psi}}_{\hat{a}}^{n a}=\tau_{\hat{a} \hat{b}}^{a b} \hat{\psi}^{n \hat{b}}{ }_{b}$. Similarly to the PPPP case, $\omega^{n m}$ (dual of $\omega^{n^{\prime} m^{\prime}}$ ) should vanish in this limit. Instead, $\tilde{\varepsilon}$ survives. So $\mathcal{N}=4$ part having supersymmetry parameter $\omega^{n m^{\prime}}$ in the original $\mathcal{N}=6$ of the ABJM theory, the additional $\mathcal{N}=2$ part with monopole operator, and the emergent 2 conformal supercharges are supercharges in the PAAP limit. As a result, the total number of supercharges in the PAAP limit is the same as in the PPPP case.

\subsection{SUSY enhancement in the PAAP limit}

Let us go back to the nonrelativistic action in the PAAP limit. In fact, the supersymmetry of the PAAP action (3.2) is much richer than the eight kinematical supersymmetry transformations 
given in (3.6). In addition, we have two more kinematical supercharges constructed from the monopole operators,

$$
\begin{aligned}
& \delta y^{n}=-\check{\xi}_{-} \tau \cdot \hat{\psi}_{+}^{n}, \quad \delta y_{n}^{\dagger}=\check{\xi}_{-}^{\dagger} \bar{\tau} \cdot \hat{\psi}_{+n}^{\dagger}, \\
& \delta \hat{y}_{n^{\prime}}=-\check{\xi}_{-}^{\dagger} \bar{\tau} \cdot \psi_{-n^{\prime}}, \quad \delta \hat{y}^{\dagger n^{\prime}}=\check{\xi}_{-} \tau \cdot \psi^{\dagger n^{\prime}} \text {, } \\
& \delta \hat{\psi}_{+}^{n}=\check{\xi}_{-} \bar{\tau} \cdot y^{n}, \quad \delta \hat{\psi}_{+n}^{\dagger}=\check{\xi}_{\tau} \cdot y_{n}^{\dagger} \text {, } \\
& \delta \psi_{-n^{\prime}}=\check{\xi}_{-} \tau \cdot \hat{y}_{n^{\prime}}, \quad \delta \psi_{-}^{\dagger n^{\prime}}=\check{\xi}_{-}^{\dagger} \bar{\tau} \cdot \hat{y}^{\dagger n^{\prime}}, \\
& \delta A_{t}=\frac{\pi \hbar}{k m}\left(\tau \cdot y_{n}^{\dagger} \check{\xi}_{-} \hat{\psi}_{+}^{n}-\check{\xi}_{-}^{\dagger} \hat{\psi}_{+n}^{\dagger} \bar{\tau} \cdot y^{n}+\check{\xi}_{-}^{\dagger} \psi_{-n^{\prime}} \bar{\tau} \cdot \hat{y}^{\dagger n^{\prime}}-\tau \cdot \hat{y}_{n^{\prime}} \check{\xi}_{-} \psi_{-}^{\dagger n^{\prime}}\right), \\
& \delta \hat{A}_{t}=\frac{\pi \hbar}{k m}\left(\check{\xi}_{-} \hat{\psi}_{-}^{n} \tau \cdot y_{n}^{\dagger}-\bar{\tau} \cdot y^{n} \check{\xi}_{-}^{\dagger} \hat{\psi}_{+n}^{\dagger},+\bar{\tau} \cdot \hat{y}^{\dagger n^{\prime}} \check{\xi}_{-}^{\dagger} \psi_{-n^{\prime}}-\check{\xi}_{-} \psi_{-}^{\dagger n^{\prime}} \tau \cdot \hat{y}_{n^{\prime}}\right), \\
& \delta A_{ \pm}=\delta \hat{A}_{ \pm}=0
\end{aligned}
$$

where $\check{\xi}_{-}$is a one-component complex spinor parameter. The action of monopole operators on the fields is expressed as

$$
\left(\tau \cdot y^{\dagger}\right)_{\hat{a}}^{a}=\tau_{\hat{a} \hat{b}}^{a b} y_{b}^{\dagger \hat{b}}, \quad(\bar{\tau} \cdot y)^{\hat{a}}{ }_{a}=\bar{\tau}_{a b}^{\hat{a} \hat{b}} y_{\hat{b}}^{b},
$$

where $y$ and $y^{\dagger}$ are in the bi-fundamental and anti-bi-fundamental representations respectively.

In addition there are two dynamical enhanced supersymmetries which are also constructed from the monopole operators,

$$
\begin{aligned}
& \delta y^{n}=\frac{i}{2 m} \check{\xi}_{+} \tau \cdot D_{-} \hat{\psi}_{+}^{n}, \quad \delta y_{n}^{\dagger}=\frac{i}{2 m} \check{\xi}_{+}^{\dagger} \bar{\tau} \cdot D_{+} \hat{\psi}_{+n}^{\dagger}, \\
& \delta y_{n^{\prime}}=\frac{i}{2 m} \check{\xi}_{+}^{\dagger} \bar{\tau} \cdot D_{+} \psi_{-n^{\prime}}, \quad \delta y^{\dagger n^{\prime}}=\frac{i}{2 m} \check{\xi}_{+} \tau \cdot D_{-} \psi_{-}^{\dagger n^{\prime}}, \\
& \delta \hat{\psi}_{+}^{n}=-\frac{i}{2 m} \check{\xi}_{+}^{\dagger} \bar{\tau} \cdot D_{+} y^{n}, \quad \delta \hat{\psi}_{+n}^{\dagger}=\frac{i}{2 m} \check{\xi}_{+} \tau \cdot D_{-} y_{n}^{\dagger}, \\
& \delta \psi_{-n^{\prime}}=-\frac{i}{2 m} \check{\xi}_{+} \tau \cdot D_{-} \hat{y}_{n^{\prime}}, \quad \delta \psi_{-}^{\dagger n^{\prime}}=\frac{i}{2 m} \check{\xi}_{+}^{\dagger} \bar{\tau} \cdot D_{+} \hat{y}^{\dagger n^{\prime}}, \\
& \delta A_{t}=\frac{i \pi \hbar}{2 k m^{2}}\left(\tau \cdot D_{-} \hat{\psi}_{+}^{n} \check{\xi}_{+} y_{n}^{\dagger}+y^{n} \bar{\tau} \cdot D_{+} \hat{\psi}_{+n}^{\dagger} \check{\xi}_{+}+\tau \cdot D_{-} \psi_{-}^{\dagger n^{\prime}} \check{\xi}_{+} \hat{y}_{n^{\prime}}+\hat{y}^{\dagger n^{\prime}} \bar{\tau} \cdot D_{+} \psi_{-n^{\prime}} \check{\xi}_{+}^{\dagger}\right) \text {, } \\
& \delta A_{+}=\frac{2 \pi}{k m}\left(\tau \cdot \hat{\psi}_{+}^{n} \check{\xi}_{+} y_{n}^{\dagger}-\tau \cdot \psi_{-}^{\dagger n^{\prime}} \check{\xi}_{+} \hat{y}_{n^{\prime}}\right), \\
& \delta A_{-}=\frac{2 \pi}{k m}\left(\hat{y}^{\dagger n^{\prime}} \bar{\tau} \cdot \psi_{-a^{\prime}} \check{\xi}_{+}^{\dagger}-y^{n} \bar{\tau} \cdot \hat{\psi}_{+n}^{\dagger} \check{\xi}_{+}^{\dagger}\right), \\
& \delta \hat{A}_{t}=\frac{i \pi \hbar}{2 k m^{2}}\left(\bar{\tau} \cdot D_{+} \hat{\psi}_{+n}^{\dagger} \check{\xi}_{+}^{\dagger} y^{n}+y_{n}^{\dagger} \tau \cdot D_{-} \hat{\psi}_{+}^{n} \check{\xi}+\bar{\tau} \cdot D_{+} \psi_{-n^{\prime}} \check{\xi}_{+}^{\dagger} \hat{y}^{\dagger n^{\prime}}+\hat{y}_{n^{\prime}} \tau \cdot D_{-} \psi_{-}^{\dagger n^{\prime}} \check{\xi}_{+}\right) \text {, } \\
& \delta \hat{A}_{+}=\frac{2 \pi}{k m}\left(y_{n}^{\dagger} \tau \cdot \hat{\psi}_{+}^{n} \check{\xi}_{+}-\hat{y}_{n^{\prime}} \tau \cdot \psi_{-}^{\dagger n^{\prime}} \check{\xi}_{+}\right), \\
& \delta \hat{A}_{-}=\frac{2 \pi}{k m}\left(\bar{\tau} \cdot \psi_{-n^{\prime}} \check{\xi}_{+}^{\dagger} \hat{y}^{\dagger n^{\prime}}-\bar{\tau} \cdot \hat{\psi}_{+n}^{\dagger} \check{\xi}_{+}^{\dagger} y^{n}\right),
\end{aligned}
$$

where $\check{\xi}_{+}$is a one-component spinor parameter. 


\subsection{Dynamical superalgebra}

In this section, we construct the supercharges coming from the transformations given in the previous subsection 4.2. Together with the $U(1)_{R} R$-symmetry mentioned in the subsubsection 3.3 .2 (its expression is given below in (4.11)) and the bosonic Schrödinger algebra (3.22), they form a dynamical superalgebra.

First, the 2 kinematical supercharges related with the transformation of (4.6) are given by

$$
\begin{aligned}
& q_{+}=-i \int d^{2} x \operatorname{tr}\left(y_{n}^{\dagger} \tau \cdot \hat{\psi}_{+}^{n}+\psi_{-}^{\dagger n^{\prime}} \tau \cdot \hat{y}_{n^{\prime}}\right), \\
& q_{-}=i \int d^{2} x \operatorname{tr}\left(\hat{y}^{\dagger n^{\prime}} \bar{\tau} \cdot \psi_{-n^{\prime}}+\hat{\psi}_{+n}^{\dagger} \bar{\tau} \cdot y^{n}\right),
\end{aligned}
$$

where $q_{-}=q_{+}^{\dagger}$. The 2 dynamical supercharges associated with the transformations given in (4.7) are obtained as

$$
\begin{aligned}
& Q_{+}=-\frac{1}{2 m} \int d^{2} x \operatorname{tr}\left(y_{n}^{\dagger} \tau \cdot D_{-} \hat{\psi}_{+}^{n}+\psi_{-}^{\dagger n^{\prime}} \tau \cdot D_{-} \hat{y}_{n^{\prime}}\right) \\
& Q_{-}=\frac{1}{2 m} \int d^{2} x \operatorname{tr}\left(\hat{y}^{\dagger n^{\prime}} \bar{\tau} \cdot D_{+} \psi_{-n^{\prime}}+\hat{\psi}_{+n}^{\dagger} \bar{\tau} \cdot D_{+} y^{n}\right),
\end{aligned}
$$

where $Q_{-}=Q_{+}^{\dagger}$. In addition, the 2 conformal supercharges which satisfy $i\left[K, Q_{ \pm}\right]=S_{ \pm}$are given by

$$
\begin{aligned}
& S_{+}=t Q_{+}+\frac{i}{2} \int d^{2} x\left(x_{1}-i x_{2}\right) \operatorname{tr}\left(y_{n}^{\dagger} \tau \cdot \hat{\psi}_{+}^{n}+\psi_{-}^{\dagger n^{\prime}} \tau \cdot \hat{y}_{n^{\prime}}\right), \\
& S_{-}=t Q_{-}-\frac{i}{2} \int d^{2} x\left(x_{1}+i x_{2}\right) \operatorname{tr}\left(\hat{y}^{\dagger n^{\prime}} \bar{\tau} \cdot \psi_{-n^{\prime}}+\hat{\psi}_{+n}^{\dagger} \bar{\tau} \cdot y^{n}\right),
\end{aligned}
$$

where $S_{-}=S_{+}^{\dagger}$. The $U(1)_{R} R$-symmetry generator is written as

$$
R=-\frac{1}{2} \int d^{2} x \operatorname{tr}\left(y_{n}^{\dagger} y^{n}+\psi_{-}^{\dagger n^{\prime}} \psi_{-n^{\prime}}-\hat{y}^{\dagger n^{\prime}} \hat{y}_{n^{\prime}}-\hat{\psi}_{+n}^{\dagger} \hat{\psi}_{+}^{n}\right) .
$$

Note that this generator represents difference between the number of particles and that of antiparticles. Recall that the total number operator (3.17) is also conserved. Therefore in our nonrelativistic limit particle and antiparticle numbers are conserved separately.

Putting everything together, we obtain the following dynamical superalgebra whose (anti)commutation relations are given by 4 :

$$
\begin{aligned}
& \left\{Q_{+}, Q_{-}\right\}=\frac{1}{2 m} H, \quad\left\{S_{+}, S_{-}\right\}=\frac{1}{2 m} K, \quad\left\{q_{+}, q_{-}\right\}=\mathcal{N}, \\
& \left\{q_{+}, Q_{-}\right\}=\frac{1}{2 m} P_{+}, \quad\left\{q_{-}, Q_{+}\right\}=\frac{1}{2 m} P_{-}, \quad\left\{q_{+}, S_{-}\right\}=-\frac{1}{2 m} G_{+}, \quad\left\{q_{-}, S_{+}\right\}=-\frac{1}{2 m} G_{-}, \\
& \left\{Q_{+}, S_{-}\right\}=\frac{1}{4 m}\left[D+i\left(J-\frac{3}{2} \tilde{R}\right)\right], \quad\left\{Q_{-}, S_{+}\right\}=\frac{1}{4 m}\left[D-i\left(J-\frac{3}{2} \tilde{R}\right)\right],
\end{aligned}
$$

\footnotetext{
${ }^{4}$ The sub-index $+(-)$ in the supercharges denotes spin up(down) state.
} 
and

$$
\begin{aligned}
& i\left[K, Q_{ \pm}\right]=S_{ \pm}, \quad i\left[G_{+}, Q_{+}\right]=-q_{+}, \quad i\left[G_{-}, Q_{-}\right]=-q_{-}, \\
& i\left[H, S_{ \pm}\right]=-Q_{ \pm}, \quad i\left[P_{+}, S_{+}\right]=-q_{+}, \quad i\left[P_{-}, S_{-}\right]=-q_{-}, \\
& i\left[D, Q_{ \pm}\right]=Q_{ \pm}, \quad i\left[D, S_{ \pm}\right]=-S_{ \pm}, \quad\left[D, q_{ \pm}\right]=0, \\
& {\left[J, Q_{ \pm}\right]=\mp \frac{1}{2} Q_{ \pm}, \quad\left[J, S_{ \pm}\right]=\mp \frac{1}{2} S_{ \pm}, \quad\left[J, q_{ \pm}\right]= \pm \frac{1}{2} q_{ \pm},}
\end{aligned}
$$

where we introduce $\tilde{R}=\frac{2}{3}(2 R+\Sigma), P_{ \pm}=P_{1} \pm i P_{2}$, and $G_{ \pm}=G_{1} \pm i G_{2}$. The commutation relations for $R$ are

$$
\begin{aligned}
& {\left[R, Q_{ \pm}\right]=\mp Q_{ \pm}, \quad\left[R, S_{ \pm}\right]=\mp S_{ \pm}, \quad\left[R, q_{ \pm}\right]=\mp q_{ \pm},} \\
& {\left[R, q_{+}^{n n^{\prime}}\right]=0, \quad\left[R, q_{-n n^{\prime}}\right]=0 .}
\end{aligned}
$$

Then the additional commutation relations can be computed as

$$
\begin{aligned}
{\left[\tilde{R}, Q_{ \pm}\right] } & =\mp Q_{ \pm}, \quad\left[\tilde{R}, S_{ \pm}\right]=\mp S_{ \pm}, \quad\left[\tilde{R}, q_{ \pm}\right]=\mp q_{ \pm} \\
{\left[\tilde{R}, q_{+}^{n n^{\prime}}\right] } & =\frac{1}{3} q_{+}^{n n^{\prime}}, \quad\left[\tilde{R}, q_{-n n^{\prime}}\right]=-\frac{1}{3} q_{-n n^{\prime}} .
\end{aligned}
$$

The super Schrödinger algebra [39, 52, 53] consists of bosonic Schrödinger algebra (3.22), kinematical superalgebra (3.25), and the dynamical superalgebra (4.12),(4.13), (4.14). This super Schrödinger algebra is isomorphic to the one in the PPPP limit [31,32].

\section{Conclusion}

In this work we considered nonrelativistic limits of the ABJM theory. In particular, we focused on the PAAP limit containing both particles and antiparticles. This case was considered previously in the literature [31]. The known result was that in this limit there are only eight kinematical supercharges. This is in contrast to the case of PPPP limit containing only particles [31, 32]. In PPPP limit one has fourteen independent supercharges: In addition to the eight kinematical supercharges there are two dynamical, two more kinematical as well as two conformal supercharges.

In this work we revisited the PAAP limit and among others we found that if we extend our considerations to supersymmetry transformations involving monopole operators, then there are six additional supercharges besides the basic eight kinematical supercharges found in [31. These additional supercharges consist of two dynamical, two kinematical, and two conformal ones.

At the same time we analyzed the PPPP limit as well and found that there is no additional supersymmetry enhancement due to monopole operator in this limit. It appears that supersymmetry in both PPPP and PAAP limit match as a number as well as a structure: ten kinematical, two 
dynamical and two conformal supercharges. In fact, both supersymmetry algebras are isomorphic to the super Schrödinger algebra with fourteen supercharges.

These results give some insight into the rôle of the monopole operator in ABJM theory. First of all the existence of additional supercharges in the nonrelativistic PAAP limit is possible because the monopole operators map antiparticle states into particle states extending the supersymmetry transformations to one mixing particles with antiparticles. This also explains why one can not observe any extended supersymmetries in the PPPP limit where there are only particles. In the nonrelativistic limit the particles and antiparticles are explicitly separated and therefore the physical meaning of the monopole operator which mixes them becomes particularly clear.

The fact that both PPPP and PAAP limits possess isomorphic supersymmetries suggests the conjecture that the super Schrödinger algebra with fourteen supercharges is the maximal unbroken supersymmetry in the nonrelativistic limit. Moreover, out of the fourteen supercharges two of them seem to be new: the conformal supercharges appear due to the emergence of nonrelativistic conformal symmetry in $c \rightarrow \infty$ limit. Therefore only twelve supersymmetries can be considered as "genuine" ones coming from the relativistic theory. This corresponds to $\mathcal{N}=6$ relativistic spinor supercharges from $\mathcal{N}=8$ total supersymmetry including the monopole enhanced supersymmetry part. The technical part of this supersymmetry breaking is clear: For either choice of nonrelativistic limit there are at least two kinds of supersymmetry transformation which are producing fields with wrong phase, therefore they should be broken. However, more fundamental reason for this breaking is not clear to us. A further study may clarify this issue.

We restricted our detailed analysis to the case of $\mathrm{U}(2) \times \mathrm{U}(2)$ gauge group, because in this case the monopole operator as well as the corresponding supersymmetry transformations can be constructed explicitly. It would be interesting, however, to apply our analysis to the enhanced supersymmetry of ABJM theory with $\mathrm{U}(N) \times \mathrm{U}(N)$ gauge symmetry in the three-algebra approach of Ref. [5]. Furthermore, these results might be generalized to any nonrelativistic limit of superconformal field theories [40, 41] which include both particles and antiparticles, and allow supersymmetries generated by the monopole operators. These aspects also deserve further investigation.

Another important subject that remains to be considered is to explore the M-theory dual of the observed phenomena. This should be of particular interest for the AdS dual description of low dimensional condensed matter systems. Let us note that the present approach to AdS/CMT correspondence is mainly based on the embedding of the nonrelativistic system into a higherdimensional relativistic one using the light-cone approach. The approach we followed here is based on the nonrelativistic reduction of a relativistic system in the same dimension which seems to be more natural since any condensed matter system is, in fact, a nonrelativistic limit of a relativistic system. 


\section{Acknowledgements}

We would like to thank Shinsuke Kawai for helpful discussions. O.K., C.S., and J.S. would like to thank the APCTP for its hospitality, during the workshop "APCTP Focus Program on Current Trends in String Field Theory", where part of this work was done. This work is supported by the National Research Foundation of Korea(NRF) grant funded by the Korea government(MEST) through the Center for Quantum Spacetime(CQUeST) of Sogang University with grant number 2005-0049409(P.O.) and the Korea Research Foundation(KRF) grant funded by the Korea government(MEST) (No. 2009-0073775) (O.K.).

\section{References}

[1] J. H. Schwarz, "Superconformal Chern-Simons theories," JHEP 0411, 078 (2004) arXiv:hep-th/0411077.

[2] J. Bagger and N. Lambert, "Modeling multiple M2's," Phys. Rev. D 75, 045020 (2007) arXiv:hep-th/0611108;

"Gauge Symmetry and Supersymmetry of Multiple M2-Branes," Phys. Rev. D 77, 065008 (2008) arXiv:0711.0955 [hep-th]];

"Comments On Multiple M2-branes," JHEP 0802, 105 (2008) [arXiv:0712.3738 [hep-th]].

[3] A. Gustavsson, "Algebraic structures on parallel M2-branes," Nucl. Phys. B 811, 66 (2009) arXiv:0709.1260 [hep-th]].

[4] O. Aharony, O. Bergman, D. L. Jafferis and J. Maldacena, "N=6 superconformal Chern-Simons-matter theories, M2-branes and their gravity duals," JHEP 0810, 091 (2008) arXiv:0806.1218 [hep-th]].

[5] A. Gustavsson and S. J. Rey, "Enhanced N=8 Supersymmetry of ABJM Theory on R(8) and $\mathrm{R}(8) / \mathrm{Z}(2)$, , arXiv:0906.3568 [hep-th].

[6] O. K. Kwon, P. Oh and J. Sohn, "Notes on Supersymmetry Enhancement of ABJM Theory," JHEP 0908, 093 (2009) [arXiv:0906.4333 [hep-th]].

[7] D. Berenstein and D. Trancanelli, "Three-dimensional N=6 SCFT's and their membrane dynamics," Phys. Rev. D 78, 106009 (2008) arXiv:0808.2503 [hep-th]].

[8] I. Klebanov, T. Klose and A. Murugan, "AdS 4 /CFT - Squashed, Stretched and Warped," JHEP 0903, 140 (2009) arXiv:0809.3773 [hep-th]].

[9] C. S. Park, "Comments on Baryon-like Operators in N=6 Chern-Simons-matter theory of ABJM," arXiv:0810.1075 [hep-th]. 
[10] Y. Imamura, "Monopole operators in N=4 Chern-Simons theories and wrapped M2-branes," Prog. Theor. Phys. 121, 1173 (2009) arXiv:0902.4173 [hep-th]].

[11] S. Kim, "The complete superconformal index for N=6 Chern-Simons theory," Nucl. Phys. B 821, 241 (2009) [arXiv:0903.4172 [hep-th]].

[12] M. M. Sheikh-Jabbari and J. Simon, "On Half-BPS States of the ABJM Theory," JHEP 0908, 073 (2009) arXiv:0904.4605 [hep-th]].

[13] D. Berenstein and J. Park, "The BPS spectrum of monopole operators in ABJM: towards a field theory description of the giant torus," arXiv:0906.3817 [hep-th].

[14] M. K. Benna, I. R. Klebanov and T. Klose, "Charges of Monopole Operators in Chern-Simons Yang-Mills Theory," arXiv:0906.3008 [hep-th].

[15] S. Kim and K. Madhu, "Aspects of monopole operators in N=6 Chern-Simons theory," JHEP 0912, 018 (2009) arXiv:0906.4751 [hep-th]].

[16] Y. Imamura and S. Yokoyama, "A Monopole Index for N=4 Chern-Simons Theories," Nucl. Phys. B 827, 183 (2010) [arXiv:0908.0988 [hep-th]].

[17] D. T. Son, "Toward an AdS/cold atoms correspondence: a geometric realization of the Schroedinger symmetry," Phys. Rev. D 78, 046003 (2008) arXiv:0804.3972 [hep-th]].

[18] K. Balasubramanian and J. McGreevy, "Gravity duals for non-relativistic CFTs," Phys. Rev. Lett. 101, 061601 (2008) [arXiv:0804.4053 [hep-th]].

[19] C. P. Herzog, M. Rangamani and S. F. Ross, "Heating up Galilean holography," JHEP 0811, 080 (2008) arXiv:0807.1099 [hep-th]].

[20] W. D. Goldberger, "AdS/CFT duality for non-relativistic field theory," JHEP 0903, 069 (2009) arXiv:0806.2867 [hep-th]].

[21] J. L. F. Barbon and C. A. Fuertes, "On the spectrum of nonrelativistic AdS/CFT," JHEP 0809, 030 (2008) arXiv:0806.3244 [hep-th]].

[22] J. Maldacena, D. Martelli and Y. Tachikawa, "Comments on string theory backgrounds with non-relativistic conformal symmetry," JHEP 0810, 072 (2008) [arXiv:0807.1100 [hep-th]].

[23] A. Adams, K. Balasubramanian and J. McGreevy, "Hot Spacetimes for Cold Atoms," JHEP 0811, 059 (2008) arXiv:0807.1111 [hep-th]].

[24] S. A. Hartnoll and K. Yoshida, "Families of IIB duals for nonrelativistic CFTs," JHEP 0812, 071 (2008) arXiv:0810.0298 [hep-th]]. 
[25] A. Donos and J. P. Gauntlett, "Supersymmetric solutions for non-relativistic holography," JHEP 0903, 138 (2009) arXiv:0901.0818 [hep-th]].

[26] A. Volovich and C. Wen, "Correlation Functions in Non-Relativistic Holography," JHEP 0905, 087 (2009) arXiv:0903.2455 [hep-th]].

[27] N. Bobev, A. Kundu and K. Pilch, "Supersymmetric IIB Solutions with Schródinger Symmetry," JHEP 0907, 107 (2009) [arXiv:0905.0673 [hep-th]].

[28] A. Donos and J. P. Gauntlett, "Schrodinger invariant solutions of type IIB with enhanced supersymmetry," JHEP 0910, 073 (2009) [arXiv:0907.1761 [hep-th]].

[29] C. Duval, G. W. Gibbons and P. Horvathy, "Celestial Mechanics, Conformal Structures, and Gravitational Waves," Phys. Rev. D 43 (1991) 3907 arXiv:hep-th/0512188.

[30] C. Duval, M. Hassaine and P. A. Horvathy, "The geometry of Schródinger symmetry in gravity background/non-relativistic CFT," Annals Phys. 324, 1158 (2009) arXiv:0809.3128 [hep-th]].

[31] Y. Nakayama, M. Sakaguchi and K. Yoshida, "Non-Relativistic M2-brane Gauge Theory and New Superconformal Algebra," JHEP 0904, 096 (2009) [arXiv:0902.2204 [hep-th]].

[32] K. M. Lee, S. Lee and S. Lee, "Nonrelativistic Superconformal M2-Brane Theory," JHEP 0909, 030 (2009) arXiv:0902.3857 [hep-th]].

[33] E. O. Colgain and H. Yavartanoo, "NR CFT duals in M-theory," JHEP 0909, 002 (2009) arXiv:0904.0588 [hep-th]].

[34] H. Ooguri and C. S. Park, "Supersymmetric non-relativistic geometries in M-theory," Nucl. Phys. B 824, 136 (2010) arXiv:0905.1954 [hep-th]].

[35] Y. Nakayama and S. J. Rey, "Observables and Correlators in Nonrelativistic ABJM Theory," JHEP 0908, 029 (2009) arXiv:0905.2940 [hep-th]].

[36] J. Jeong, H. C. Kim, S. Lee, E. O. Colgain and H. Yavartanoo, "Schrodinger invariant solutions of M-theory with Enhanced Supersymmetry," arXiv:0911.5281 [hep-th].

[37] S. Kawai and S. Sasaki, "BPS Vortices in Non-relativistic M2-brane Chern-Simons-matter Theory," Phys. Rev. D 80, 025007 (2009) [arXiv:0903.3223 [hep-th]].

[38] C. R. Hagen, "Scale and conformal transformations in galilean-covariant field theory," Phys. Rev. D 5, 377 (1972);

U. Niederer, "The maximal kinematical invariance group of the free Schrodinger equation," Helv. Phys. Acta 45 (1972) 802. 
[39] M. Leblanc, G. Lozano and H. Min, "Extended superconformal Galilean symmetry in Chern-Simons matter systems," Annals Phys. 219, 328 (1992) arXiv:hep-th/9206039.

[40] Y. Nakayama, "Index for Non-relativistic Superconformal Field Theories," JHEP 0810, 083 (2008) arXiv:0807.3344 [hep-th]].

[41] Y. Nakayama, S. Ryu, M. Sakaguchi and K. Yoshida, "A family of super Schrodinger invariant Chern-Simons matter systems," JHEP 0901, 006 (2009) arXiv:0811.2461 [hep-th]].

[42] Y. Nakayama and M. Sakaguchi, "Interacting SUSY-singlet matter in non-relativistic Chern-Simons theory," J. Phys. A 42, 195402 (2009) arXiv:0812.1564 [hep-th]].

[43] K. Hosomichi, K. M. Lee, S. Lee, S. Lee and J. Park, "N=5,6 Superconformal Chern-Simons Theories and M2-branes on Orbifolds," JHEP 0809, 002 (2008) arXiv:0806.4977 [hep-th]].

[44] J. Gomis, D. Rodriguez-Gomez, M. Van Raamsdonk and H. Verlinde, "A Massive Study of M2-brane Proposals," JHEP 0809, 113 (2008) [arXiv:0807.1074 [hep-th]].

[45] M. Benna, I. Klebanov, T. Klose and M. Smedback, "Superconformal Chern-Simons Theories and $\mathrm{AdS}_{4} / \mathrm{CFT}_{3}$ Correspondence," JHEP 0809, 072 (2008) arXiv:0806.1519 [hep-th]].

[46] J. Gomis, A. J. Salim and F. Passerini, "Matrix Theory of Type IIB Plane Wave from Membranes," JHEP 0808, 002 (2008) [arXiv:0804.2186 [hep-th]].

[47] K. Hosomichi, K. M. Lee and S. Lee, "Mass-Deformed Bagger-Lambert Theory and its BPS Objects," Phys. Rev. D 78, 066015 (2008) [arXiv:0804.2519 [hep-th]].

[48] C. Kim, Y. Kim, O. K. Kwon and H. Nakajima, "Vortex-type Half-BPS Solitons in ABJM Theory," Phys. Rev. D 80, 045013 (2009) [arXiv:0905.1759 [hep-th]].

[49] Y. Kim, O. K. Kwon, H. Nakajima and D. D. Tolla, "Coupling between M2-branes and Form Fields," JHEP 0910, 022 (2009) [arXiv:0905.4840 [hep-th]].

[50] N. Lambert and P. Richmond, "M2-Branes and Background Fields," JHEP 0910, 084 (2009) arXiv:0908.2896 [hep-th]].

[51] R. Jackiw and S. Y. Pi, "Classical and quantal nonrelativistic Chern-Simons theory," Phys. Rev. D 42, 3500 (1990) [Erratum-ibid. D 48, 3929 (1993)].

[52] C. Duval and P. A. Horvathy, "On Schrodinger superalgebras," J. Math. Phys. 35, 2516 (1994) arXiv:hep-th/0508079. 
[53] M. Sakaguchi and K. Yoshida, "Super Schrodinger in Super Conformal," J. Math. Phys. 49, 102302 (2008) arXiv:0805.2661 [hep-th]];

"More super Schrodinger algebras from psu(2,2|4)," JHEP 0808, 049 (2008) arXiv:0806.3612 [hep-th]]. 